Onchocerciasis

\section{The order is rapidly fadin'}

\section{J D Chidambaram, T M Lietman}

\section{Onchocerciasis and trachoma may become historical diseases within our lifetime}

In this issue of the BJO (p 796), Egbert et al utilise a case-control design to demonstrate that onchocerciasis and glaucoma are associated in an area of Ghana. Perhaps this is not surprising, as onchocerciasis is known to cause anterior segment inflammation and peripheral anterior synechiae, which can in turn lead to increased intraocular pressure. However, this association had never really been proved in the past, in part because reliable glaucoma data in the developing world have been difficult to come by. Interestingly, as glaucoma has become more recognised, onchocerciasis has become less so-glaucoma has moved up to number two in the WHO's latest rankings, while onchocerciasis may have made the list for the last time at number 8 (table 1). ${ }^{1}$ This may be the ideal time to make such an association between these two diseases when awareness of both diseases is relatively high-it would have been difficult in the past or the future.

Many factors go into the disease rankings. To some extent, they reflect the efficacy of treatment. Programmes have had remarkable success with those diseases amenable to mass drug distributions (neonatal ophthalmia, xerophthalmia, onchocerciasis, and trachoma). In fact, onchocerciasis and trachoma may become historical diseases within our lifetime. As developing countries become wealthier with improved diet and longer life expectancies, we expect worldwide rankings to reflect diseases now found often in the developed countries. Worldwide, diabetes is increasing at an alarming rate, and diabetic retinopathy is now fifth on the list. Older populations are more susceptible to age related macular degeneration (AMD), now third. ${ }^{1}$ Both diabetic retinopathy and AMD had not even appeared on previous rankings. ${ }^{2}$ This echoes what has been previously been noted with mortality statistics, where projected worldwide rankings are similar to current rankings in developed countries. ${ }^{3}$ Difficulties in the diagnosis of disease also come into play. Some diseases are easily found on the external examination (trachoma), or at least with an undilated examination (mature cataract). Others, such as glaucoma, require equipment and dilated examination-the harder people look, the more glaucoma they find. Glaucoma moved up to third in the $1990 \mathrm{~s}^{2}$ and to second in the most recent survey, ${ }^{1}$ in part the result of better diagnosis. There are also fluctuations in the awareness and politics of disease. More than one country has been reluctant to attribute blindness to trachoma, since it had been declared eliminated in the past. To some extent, the changes in rankings reflect secular, socioeconomic trends. Trachoma is disappearing in much of the world, even in the absence of programmes specifically targeting the disease. This may be the

Table 1 Global blindness rankings for 1994 and 2002

\begin{tabular}{llr}
\hline & Cause & $\%$ \\
\hline 1994 ranking* $_{1}$ & & \\
2 & Cataract & 41.8 \\
3 & Trachoma & 15.5 \\
4 & Glaucoma & 13.5 \\
5 & Vitamin A deficiency & 1.3 \\
6 & Trauma & 1.3 \\
7 & Onchocerciasis & 0.9 \\
2002 ranking* & Leprosy & 0.7 \\
1 & Cataract & 47.8 \\
2 & Glaucoma & 12.3 \\
3 & Age related macular degeneration & 8.7 \\
4 & Corneal opacities & 5.1 \\
5 & Diabetic retinopathy & 4.8 \\
6 & Childhood blindness & 3.9 \\
7 & Trachoma & 3.6 \\
8 & Onchocerciasis & 0.8 \\
\hline \multirow{2}{*}{ *Adapted from references 1 and 2. } & \\
\hline
\end{tabular}

result of better hygiene, fewer flies, and perhaps even widespread use of antibiotics for other purposes that incidentally cover chlamydia. ${ }^{4}$ The rankings also reflect the vagaries of estimation. In the past decade trachoma has gone from second to seventh in the rankings, in part because of mass treatment programmes, the presence of a secular trend, and the realisation that previous estimates of the burden of trachomatous blindness were just too high.

Associations between diseases and rankings of their importance worldwide are not just curiosities. They can be of great practical importance. Programmes targeting onchocerciasis, trachoma, lymphatic filariasis, and schistosomiasis all distribute antimicrobials to large segments of the population. Groups are beginning to study how the geographical distributions of these infections overlap. Immunisation campaigns have already demonstrated that mass administrations can be used for the delivery of other preventive health services such as vitamin A distribution. Synergy in surgical programmes may exist as well; for example, trachoma programmes often pick up more mature cataracts than trichiasis. As programmes expand, it will be important to integrate, so as not to overburden public health programmes with limited resources. Just as important is not to overburden rural, subsistence farmers with requests to attend separate onchocerciasis days, trachoma days, polio days, etc. The rankings of the major causes of blindness not only appeal to our love of lists, but also help to set priorities and demonstrate our long term successes and failures. The recent rankings may serve to alert the international community that not enough is being done for management of some diseases. Diabetes and AMD researchers can be forgiven if they tout the newly recognised importance of their diseases in papers and grant applications. Likewise onchocerciasis and trachoma programmes can be forgiven if they take some of the credit for the decline of their diseases, and now brag that they are number 8 .

Br J Ophthalmol 2005;89:789-790. doi: $10.1136 /$ bjo.2005.065847

\section{Authors' affiliations}

J D Chidambaram, T M Lietman, FI Proctor Foundation, University of California, San Francisco, CA, USA

T M Lietman, Institute for Global Health, and Department of Ophthalmology, University of California, San Francisco, CA, USA

Correspondence to: $\mathrm{Dr}$ Thomas M Lietman, FI Proctor Foundation Room 307, 95 Kirkham Street, University of California San Francisco, San Francisco, CA 94143-0944, USA; tml@ itsa.ucsf.edu 


\section{REFERENCES}

1 Resnikoff S, Pascolini D, Etya'ale D, et al. Global data on visual impairment in the year 2002. Bull World Health Organ 2004;82:844-51.
2 Thylefors B, Négrel AD, Pararajasegaram R, et al. Global data on blindness. Bull World Health Organ 1995;73:115-21.

3 Murray CJL, Lopez AD, eds. The global burden of disease: a comprehensive assessment of mortality and disability from diseases, injuries, and risk factors in 1990 and projected to 2020 Boston: Harvard University Press, 1996

4 Chidambaram JD, Bird M, Schiedler V, et al. Trachoma decline and widespread use of antimicrobial drugs. Emerg Infect Dis 2004; 10: 1895-9.

Indocyanine green

\section{Let green lead not astray}

\section{J Sebag}

\section{Trepidations of ICG use in macular hole surgery}

A t the close of the 20th century the medical world was witness to a most remarkable advance when therapy was developed for a previously incurable disease. Macular hole surgery is one of the great success stories in ophthalmology, if not modern medicine. Predicated upon an increased awareness of the role of vitreous in the pathogenesis of retinal disorders, this achievement results from the work of pioneering surgeons who had the ingenuity and courage to devise and attempt a new surgical approach that now restores vision to many grateful patients. However, recent modifications of the surgical technique may jeopardise visual outcomes and "lead us astray" from early successes.

Vitreous is an extended extracellular matrix, whose molecular composition and supramolecular organisation result in a clear gel that firmly adheres to the retina in youth. ${ }^{1-3}$ Ageing induces liquefaction and vitreoretinal dehiscence, which occur concurrently in the overwhelming majority of individuals, resulting in innocuous posterior vitreous detachment (PVD). ${ }^{4}$ Anomalous PVD ${ }^{5}$ results from vitreous liquefaction without sufficient vitreoretinal dehiscence. This may preclude the posterior vitreous cortex from separating cleanly from the internal limiting lamina (ILL) of the retina. The untoward consequences of anomalous PVD vary depending upon where in the vitreous body the gel is most liquefied and where on the retina there is greatest vitreous adherence. ${ }^{5}$ Anomalous PVD in the periphery, for example, results in retinal tears. Along blood vessels, liquefaction without vitreoretinal dehiscence induces vitreous haemorrhage. Another important effect of anomalous PVD is vitreoschisis, a split in the posterior vitreous cortex that has been identified with biomicroscopy, ${ }^{6}$ ultrasonography, ${ }^{7}$ and optical coherence tomography. ${ }^{8}$ Vitreoschisis has been confirmed by histopathological studies ${ }^{9}$ and has also been documented during surgery by intravitreal triamcinolone injection. ${ }^{10}$

Anomalous PVD is hypothesised to have a role in the pathogenesis of macular holes via vitreoschisis. ${ }^{511} 12$ Studies have identified premacular membranes by histopathology in $73 \%$ of cases $(n=22)^{13}$ and by stereoscopic fundus photography in $65 \%$ of eyes $(n=224) .{ }^{14}$ The origin of this membrane is postulated to be the outer wall of a vitreoschisis cavity in the posterior vitreous cortex, since this tissue has been identified as prefoveal vitreous by histopathology. ${ }^{15}$ In a recent clinical study ${ }^{16}$ of 69 cases of macular hole, optical coherence tomography detected a prefoveal membrane that could be an advanced form of the outer wall of a posterior vitreoschisis cavity. Migration of cells from the retina, such as fibrous astrocytes and Mueller cells, and recruitment of cells from the circulatory system by hyalocytes result in some degree of cellularity. New collagen (type I) synthesis further alters the appearance of this tissue that, when it began as the outer wall of a vitreoschisis cavity, was thin, composed primarily of type II collagen, and only contained hyalocytes. Centrifugal (outward from the fovea) traction forces are induced by the detached vitreous body, which is still attached to the peripheral circumference of the vitreoschisis cavity, where the inner and outer walls fuse into an intact posterior vitreous cortex. The extent of this anomalous PVD from the fovea has been shown to correlate with the stage of macular hole-that is, stage 2 holes have a less extensive PVD than stage 4 holes. ${ }^{17}$ Traction by this tissue is augmented by various contractile cells, especially myofibroblasts, ${ }^{14}$ resulting in a dehiscence of the central macula. ${ }^{5}$ The importance of the posterior aspect of the posterior vitreous cortex in the pathogenesis of macular holes is underscored by the salubrious outcome when this tissue is successfully removed in its entirety.

And missing thee, I walk unseen On the dry smooth-shaven green... Like one that had been led astray Through the heav'n's wide pathless way...

John Milton, II Penseroso [1631]

Although previously praised in print, ${ }^{18}$ the seminal contributions of Kelly and Wendell ${ }^{19}$ to the treatment of macular holes cannot be overemphasised, as their pioneering work paved the way for the relatively high success rate experienced by many patients. The outer wall of the vitreoschisis cavity is usually "unseen" in their procedure until it is elevated off the retinal surface. While vitreous invisibility ${ }^{3}$ is critical to its physiological function, ${ }^{1-3}$ this poses challenges for clinical imaging. ${ }^{20}$ Echography and optical coherence tomography often fail to identify the outer layer of a vitreoschisis cavity because it is usually thinner than the level of resolution of these techniques. In an attempt to assure that the "unseen" pathogenic tissues are removed and thereby increase the rate of hole closure, surgeons began to dissect farther posteriorly and tried to remove what was thought to represent the ILL. The results from one large retrospective study, ${ }^{21}$ comparing no ILL peel in 417 cases with ILL peeling in 175 cases found that ILL peeling increased the initial closure rate from $81 \%$ to $92 \%$ and decreased the reopening rate from $7 \%$ to $0.6 \%$. However, there did not appear to be any difference in visual outcomes when the hole was closed by either technique. Complete ILL removal would damage Mueller cells and negatively impact upon retinal neurophysiology and vision. Thus, it is highly unlikely that the entire ILL is removed in patients who experience improved vision. Rather, the deeper dissection undertaken during attempted ILL removal most probably creates a surgical plane between the three laminae of the 
$\mathrm{ILL}^{22}$ leaving the innermost layer, the lamina rara externa, intact and the underlying neural retina undamaged. In surgery, however, it is often difficult to accurately assess whether the ILL is being removed in part, in total, or at all.

Intraoperative efforts to enhance the visualisation of pathogenic tissues in macular holes led to the use of indocyanine green (ICG) dye to stain the tissue. Unfortunately, this was undertaken without any preclinical studies to determine safety and efficacy. Thus, while this "smooth-shaven green" approach did increase the rate of hole closure, it was associated with untoward effects on postoperative visual acuity. ${ }^{23-25}$ That ICG was the cause of poor visual acuity in spite of hole closure was, to a degree, substantiated in a subsequent study where one of these same surgeons found that a short exposure to a lower dose of ICG was associated with improved visual acuity. ${ }^{26}$ Why ICG was not found to be associated with poor postoperative visual acuity in other series $^{27}$ may relate to differences in surgical technique that probably employed a more shallow plane of dissection. However, a true understanding of these discrepant findings requires a better understanding of the mechanism of ICG toxicity.

ICG may have untoward effects via several mechanisms that are not mutually exclusive. As alluded to above, the use of ICG could result in a deeper surgical plane of dissection with damage to neural retinal elements. Histopathological analysis of tissues removed at surgery support this postulate. ${ }^{28}$ There may a direct toxic effect upon retinal neurons by a chemical interaction. In postmortem human eyes, ICG alone was associated with rupture of Mueller cells and detachment of the ILL. ${ }^{29}$ Apoptosis was induced in human RPE cells in culture with ICG. ${ }^{30}$ Since ICG is a photosensitiser, there is potential for light toxicity via a photodynamic effect. Studies ${ }^{31}$ have shown that in the presence of ICG, light from a standard endoilluminator has a dose dependent toxicity on retinal ganglion cells in vitro. Experiments in postmortem human eyes identified wavelengths longer than $620 \mathrm{~nm}$ as phototoxic, determined by light and electron microscopy. ${ }^{29}$ However, other postmortem studies ${ }^{32}$ in pig eyes found no such effects. The results of postmortem studies are often difficult to interpret, however, as they sometimes lead "through the heav'n's wide pathless way," and thus in vivo experimentation is needed to properly address this issue.

In this issue of the BJO ( $\mathrm{p}$ 897) Kwok and associates in Hong Kong report the results of in vivo studies on the effects of
ICG plus endoillumination in rabbits, assessed by electroretinography (preoperatively and postoperatively) and histopathological analyses. At 1 week after surgery, there was significant reduction in the light-adapted a-wave amplitude and significant delays in the light and dark adapted b-wave latencies. Histopathological findings included focal loss of photoreceptor outer segments, some foci of photoreceptor absence, focal oedema of the inner and outer nuclear layers, and localised areas of RPE irregularities. In the absence of a retinal break, it is surprising to find RPE and outer retinal abnormalities. Since these findings were focal in distribution, the abnormalities may have resulted from mechanical trauma (retinal elevation off the RPE) during the experimental surgery. It is well known that the rabbit vitreous is very firmly adherent to the retina. Subsequent studies must rule out any mechanical effects that might have been induced during dissection of the posterior vitreous cortex off this very adherent interface. One possible solution would be to undertake pharmacological vitreoly$\operatorname{sis}^{33} 34$ with agents intended to lyse the vitreoretinal interface, making dissection of the posterior vitreous cortex easier with less traction upon the retina. However, as these enzymes might introduce other effects, perhaps even artefacts, it would be simpler to employ a species with less adhesion at the vitreoretinal interface, such as the mini-pig, whose vitreoretinal interface more closely resembles that of humans.

The authors are to be thanked for contributing to our understanding of the effects of ICG upon retinal physiology and structure. As their studies were conducted in the absence of a retinal hole, the findings may also help interpret the observations of ICG toxicity in surgery for macular pucker ${ }^{35}$ and diabetic macular oedema. ${ }^{36}$

Br J Ophthalmol 2005;89:790-792.

doi: 10.1136/bjo.2005.065821

Correspondence to: J Sebag, MD, FACS FRCOphth, Doheny Eye Institute, University of Southern California, and VMR Institute, 7677 Center Avenue, suite 400, Huntington Beach, CA 92647, USA; jsebag@VMRinstitute.com

\section{REFERENCES}

1 Sebag J. The vitreous - structure, function, and pathobiology. New York: Springer-Verlag, 1989.

2 Sebag J. The vitreous. In: Hart WM Jr, ed. In: Adler's physiology of the eye, ed. St Louis: Mosby, 1992:268-347.

3 Sebag J. Vitreous-from biochemistry to clinical relevance. In: Tasman W, Jaeger EA, eds. In: Duane's foundations of clinical ophthalmology. Philadelphia: Lippincott Williams \& Wilkins, 2005, Vol 1, Ch 16

4 Sebag J. Classifying posterior vitreous detachment-a new way to look at the invisible. Br J Ophthalmol 1997;81:521-2.
5 Sebag J. Anomalous PVD-a unifying concept in vitreo-retinal diseases. Graefes Arch Clin Exp Ophthalmol 2004;242:690-8.

6 Kakehashi A, Schepens CL, de Sousa-Neto A, et al. Biomicroscopic findings of posterior vitreoschisis. Ophthalmic Surg 1993;24:846-50.

7 Chu TG, Lopez P, Cano MR, et al. Posterior vitreoschisis. An echographic finding in proliferative diabetic retinopathy. Ophthalmology $1996 ; 103: 315-22$

8 Carpineto P, Ciancaglini M, Aharrh-Gnama A, et al. Optical coherence tomography imaging of surgical resolution of bilateral vitreomacular traction syndrome related to incomplete posterior vitreoschisis. Eur J Ophthalmol 2004;14:438-41.

9 Schwartz SD, Alexander R, Hiscott P, et al. Recognition of vitreoschisis in proliferative diabetic retinopathy. A useful landmark in vitrectomy for diabetic traction retinal detachment. Ophthalmology 1996;103:323-8.

10 Sonoda KH, Sakamoto T, Enaida H, et al. Residual vitreous cortex after surgical posterior vitreous separation visualized by intravitreous triamcinolone acetonide. Ophthalmology 2004; 111:226-30

11 Sebag J. Vitreous anatomy and vitreo-macular interface. In: Madreperla S, McCuen B, eds. In: Macular hole-pathogenesis, diagnosis, and treatment. Woburn, MA: ButterworthHeinemann, 1999:1-24.

12 Green WR, Sebag J. Vitreous and the vitreoretinal interface. In: Ryan SJ, ed. In: Retina. St Louis: Mosby, 2001; Vol, III: 1882-960.

13 Guyer DR, Green WR, de Bustros S, et al. Histopathologic features of idiopathic macular holes and cysts. Ophthalmology 1990;97:1045-51.

14 Cheng L, Freeman WR, Ozerdem U, et al. Prevalence, correlates, and natural history of epiretinal membranes surrounding idiopathic macular holes. Ophthalmology 2000; 107:853-9.

15 Yooh HS, Brooks HL Jr, Capone A Jr, et al. Itrastructural features of tissue removed during idiopathic macular hole surgery. Am J Ophthalmol 1996;122:67-75.

16 Mizushima T, Uemura A, Sakamoto T. Prefoveolar membrane in macular hole opercula formation. Jpn J Ophthalmol 2004;48:478-85.

17 Ito $Y$, Terasaki H, Suzuki T, et al. Mapping posterior vitreous detachment by optical coherence tomography in eyes with idiopathic macular hole. Am J Ophthalmol 2003;13:351-5.

18 Sebag J. ICG-assisted macular hole surgery-too pioneering? (Guest editorial) Am J Ophthalmol 2004; 137:744-6.

19 Kelly N, Wendel RT. Vitreous surgery for idiopathic macular holes. Results of a pilot study. Arch Ophthalmol 1991;109:654-9.

20 Sebag J. Seeing the invisible: the challenge of imaging vitreous. J Biomed Opt 2004;9:38-46.

21 Kumagai K, Furukawa M, Ogino N, et al. Vitreous surgery with and without internal limiting membrane peeling for macular hole repair. Retina 2004;24:721-7.

22 Sebag J. Hageman GS. Interfaces. Eur J Ophthalmol 2000;10:1-3.

23 Haritoglou C, Gandorfer A, Gass CA, et al. Indocyanine green-assisted peeling of the internal limiting membrane in macular hole surgery affects visual outcome: a clinicopathologic correlation. Am J Ophthalmol 2002; 134:836-41.

24 Gass CA, Haritoglou C, Schaumberger M, et al. Functional outcome of macular hole surgery with and without indocyanine green-assisted peeling of the internal limiting membrane. Graefes Arch Clin Exp Ophthalmol 2003;241:716-20.

25 Ando F, Sasano K, Ohba N, et al. Anatomic and visual outcomes after indocyanine green-assisted peeling of the retinal internal limiting membrane in idiopathic macular hole surgery. Am J Ophthalmol 2004;137:609-14.

26 Ando F, Sasano K, Suzuki F, et al. Indocyanine green-assisted ILM peeling in macular hole surgery revisited. Am J Ophthalmol 2004; 138:886-7.

27 Da Mata AP, Burk SE, Foster RE, et al. Long-term follow-up of indocyanine green-assisted peeling of the retinal internal limiting membrane during vitrectomy surgery for idiopathic macular hole repair. Ophthalmology 2004;1 11:2246-53. 
28 Gandorfer A, Haritoglou C, Gass CA, et al. Indocyanine green-assisted peeling of the internal limiting membrane may cause retinal damage. Am J Ophthalmol $2001 ; 132: 431-3$

29 Gandorfer A, Haritoglou C, Gandorfer A, et al. Retinal damage from indocyanine green in experimental macular surgery. Invest Ophthalmol Vis Sci 2003:44:316-23.

30 Rezai KA, Farrokh-Siar L, Ernest JT, et al. Indocyanine green induces apoptosis in human retinal pigment epithelial cells. Am J Ophthalmol 2004; 137:931-3.

31 Iriyama A, Uchida S, Yanagi Y, et al. Effects of indocyanine green on retinal ganglion cells. Invest Ophthalmol Vis Sci 2004;45:282-6.

32 Grisanti S, Szurman P, Gelisken F, et al. Histological findings in experimental macular surgery with indocyanine green. Invest Ophthalmol Vis Sci 2004;45:282-6.

33 Sebag J. Pharmacologic vitreolysis. (Guest editorial) Retina 1998;18:1-3.
34 Sebag J. Is Pharmacologic vitreolysis brewing? (Guest editorial) Retina, 2002;22:1-3.

35 Haritoglou C, Gandorfer A, Gass CA, et al. The effect of indocyanine-green on functional outcome of macular pucker surgery. Am J Ophthalmol 2003;135:328-37.

36 Ando F, Yasui O, Hirose H, et al. Optic nerve atrophy after vitrectomy with indocyanine greenassisted internal limiting membrane peel in diffuse macular edema. Graefes Arch Clin Exp Ophthalmol 2004;242:995-9.

\section{Vision restoration therapy: confounded by eye movements}

\section{J C Horton}

\section{Treatment claims not supported by data}

$\mathrm{R}$ ecently Sabel, Kenkel, and Kasten co-authored a report showing that vision restoration therapy does not improve field defects in patients with cortical lesions. ${ }^{1}$ This finding was a disappointment because it dashed hopes that vision restoration therapy might benefit patients who suffer visual field loss from stroke, tumour, or trauma involving the occipital lobe. In a new twist, Sabel and colleagues have now written an editorial stating that "we have no objections to the data as

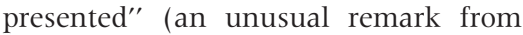
the co-authors of a study), followed in the next breath by a long argument repudiating the main thrust of their report. $^{2}$ If readers are confused, they are not to blame.

Vision restoration therapy was described previously in a series of papers by Sabel and colleagues. ${ }^{3-8}$ In brief, it attempts to restore visual field defects by having patients practise perimetry every day at home using a software package loaded onto their personal computers. The idea is that repeated visual stimulation, especially just inside a scotoma boundary, can salvage neurons in damaged cortex at the fringe of a lesion. Sabel's previous studies suffered from a major flaw: eye movements were not recorded or controlled. Patients with homonymous field loss often compensate by making surveillance saccades into their blind hemifield. Although Sabel and colleagues used the blind spot position to monitor fixation, they never reported fixation losses, false positives, or false negatives in their papers. Moreover, the blind spot position is an imperfect method for detecting small saccades, and useless for fixation assessment in an eye with a temporal hemianopia. For these reasons, most neuro-ophthalmologists were sceptical of Sabel's claims for vision restoration therapy.

To his credit, Sabel responded by undertaking a collaborative study with scientists employing the scanning laser ophthalmoscope. ${ }^{1}$ This instrument allows one to present stimuli while monitoring fixation with great precision. Trials in which the patient sneaks a saccade can be discarded, solving the problem of fixation instability. Under these testing conditions, Sabel and coworkers found no improvement in the visual fields after vision restoration therapy.

Sabel had hoped that proper monitoring of fixation with the scanning laser ophthalmoscope would vindicate vision restoration therapy. Instead, he saw its apparent therapeutic benefit evaporate once the artefact of eye movements was eliminated. Rather than accept this negative outcome, he has written a commentary defending vision restoration therapy and criticising the methods used in his own paper. ${ }^{2}$ This is worthy of further comment, if only to highlight the inconsistencies behind this about face.

Sabel's rebuttal relies on a paper in press elsewhere, showing that the same patients who failed to show improvement with the scanning laser ophthalmoscope did improve when tested with Tübingen automated perimetry and high resolution perimetry. ${ }^{9}$ It is difficult to comment on a paper that is still unpublished, but it should be recalled that Sabel has reported previously that patients with homonymous field loss do not improve after vision restoration therapy when testing is done with Tübingen automated perimetry. ${ }^{3}$ Once again, Sabel has placed himself in the position of refuting his own work. High resolution perimety refers to the technique used by Sabel to measure the visual fields before and after vision restoration therapy. ${ }^{3}$ Its drawback, as mentioned earlier, is poor control over eye movements. This deficiency was the reason for turning to the scanning laser ophthalmoscope in the first place.

Sabel's data will remain uninterpretable until he adopts a technique that eliminates the artefact created by small saccades

In his editorial, Sabel reproduces a figure from his upcoming paper, comparing the fields before and after vision restoration therapy in a patient with a hemianopia. $^{2}$ Before treatment, the scanning laser ophthalmoscope shows a field cut that deviates only about half a degree from the vertical meridian. This reflects the excellent control of eye movements afforded by that technique. The fields plotted by Tübingen automated perimetry and high resolution perimetry deviate by $1^{\circ}-8^{\circ}$ from the vertical meridian, yet they are measuring the same field defect defined by scanning laser ophthalmoscope, the gold standard. Immediately, this discrepancy should raise a warning flag. How can one define an improvement equal to only $2.5^{\circ}-3.5^{\circ}$ degrees azimuth, when one's baseline measurement of the field defect is inaccurate by this amount? Sabel's data will remain uninterpretable until he adopts a technique that eliminates the artefact created by small saccades. Parenthetically, it should be pointed out that the figure shows an unexplained discrepancy between Tübingen automated perimetry and high resolution perimetry in the elevation of the field defect before treatment.

After vision restoration therapy, Sabel shows a thin sliver of residual field loss that hugs the horizontal meridian, extending no further from the vertical meridian than it did before treatment. He asserts that such a change following treatment could not be produced by 
undetected horizontal saccades. In this single anecdotal case he is correct, but why rely on such an indirect, inferential approach to deal with the problem of fixation control? And in this particular case, why did the field cut improve dramatically in elevation but not azimuth? Anyone familiar with the visual field map in striate cortex will be puzzled by the pattern of field improvement attributed to vision restoration therapy in this case.

Sabel asserts that field testing using the scanning laser ophthalmoscope was difficult for patients, preventing vision restoration therapy from showing any benefit. It is true that patients had to report verbally their perception of three vertically aligned targets, rather than simply push a buzzer. ${ }^{1}$ However, there is no evidence that this requirement made their task more difficult or that it led to selective inaccuracy in the post-treatment assessment of their visual fields. It is also true that the scanning laser ophthalmoscope targets were dark against a bright background, to avoid light scatter. Sabel states that "Simultaneous stimulus discrimination and detection of negative stimuli on a bright background are probably tasks beyond the abilities of a damaged visual system." In fact, cells in the visual cortex respond overall equally well to stimuli that are dark, rather than light, compared to background. The retina contains approximately equal numbers of on-centre and off-centre cells, and it is no harder for a subject to detect a dark spot than a light spot, provided the contrast is high.

The stimuli used with the scanning laser ophthalmoscope were large $\left(0.33^{\circ}\right)$ and high contrast, chosen deliberately to detect absolute scotomas (much like the V4e stimulus of the Goldmann perimeter). Sabel states that "The SLO method appears to be insensitive to relative defects describing areas with residual function as being absolutely blind." ${ }^{2}$ In fact, the opposite is true. A technique that measures only absolute defects will characterise relative defects as normal. Sabel argues that the scanning laser ophthalmoscope missed regions of relative field depression that might have improved from vision restoration therapy. He forgets that with a cortical lesion, the first indication of recovery is provided by shrinkage of the absolute portion of the scotoma, even while the relative portion persists. To draw again an analogy with Goldmann perimetry, the V4e isoptre will sometimes expand in a recovering field defect, whereas the I2e isoptre will continue to show a defect. Thus, a technique that defines the patient's absolute scotoma is the most sensitive to any potential improvement.
Sabel et al write that "Horton is concerned that vision restoration therapy improvements may simply be a result of placebo effect." 2 That is not quite an accurate paraphrase of my position. In my editorial, I noted that patients had the subjective impression that they had benefited from visual restoration therapy, despite lack of improvement in their fields. ${ }^{10}$ I attributed this discrepancy between negative field results and positive patient perception to a placebo effect. I expressed concern about using patient satisfaction as an outcome criterion, because patients will clamour for a treatment they believe works, even if it's humbug.

Although neuroplasticity is active in many regions of the brain, this fact does not mean that vision restoration therapy can promote visual field recovery following lesions of the striate cortex. Sabel notes that "normal adult subjects are capable of perceptual learning, and there is an entire body of evidence on activity dependent use and neuroplasticity, such as studies on adult receptive field expansions following retinal or brain lesions." ${ }^{2}$ These statements are true, yet when examined closely they are irrelevant to Sabel's position.

Perceptual learning refers to the improvement in psychophysical performance that comes with practice. For example, anyone who takes a computerised visual field test a few times will show a slight improvement in retinal sensitivity. This phenomenon is well known, and must be taken into account when assessing the response to any proposed therapy, such as pressure lowering medications in glaucoma. ${ }^{11}$ For vision restoration therapy, perceptual learning is a confounding factor that must be controlled for by incorporating a placebo arm into studies. All subjects show a slight degree of improvement with practice, whether they have had vision restoration therapy or not.

Several investigators have reported that after retinal lesions (Sabel mistakenly refers to retinal or brain lesions), cells in the visual cortex become responsive to stimulation just outside the zone of retinal damage. ${ }^{12} 13$ The visual field does not improve, nor does the brain suffer a direct lesion. Individual cortical cells undergo expansion of their receptive fields, without any special therapy, simply because they have lost their normal input. Sabel advocates vision restoration therapy for an utterly different scenario: the restoration of lost visual field after a lesion that has injured the brain.

Sabel reminds us that the visual system is not purely sensory, because "it utilises many cognitive mechanisms as seen, for example, in the phenomenon of physiological blindspot "filling in. ${ }^{\prime 2}$ It is unclear how the ability of the visual system to fill in blind areas is relevant here. Such areas remain blind, and subjects cannot detect visual stimulation. The fill-in phenomenon has nothing to do with the concept behind vision restoration therapy, and makes it no more plausible.

Sabel states that "the Food and Drug Administration has cleared vision restoration therapy to be offered in the United States and has done so in recognition of the results from the Tübingen-Magdeburg trial. ${ }^{\prime 2}$ It is true that the FDA granted a $510(\mathrm{k})$ clearance to NovaVision's vision restoration therapy on 22 April 2003, in response to an application filed on 25 October 2002. A $510(\mathrm{k})$ clearance is required before marketing certain types of new medical devices in the United States. The applicant must demonstrate that the device is "substantially equivalent" to a legally marketed device introduced previously. In this case, the predicate device was DynaVision 2000, a similar program for treating amblyopia. A $510(\mathrm{k})$ clearance is not based on recognition of the results of a clinical trial, and Sabel's application to the FDA did not rely on the Tübingen-Magdeburg trial. (Readers can judge the veracity of Sabel's statement by inspecting his application at www.fda.gov/cdrh/pdf2/k023623.pdf)

Sabel asserts that "several clinical centres throughout the United States are now beginning to observe similar improvements with their first patients." ${ }^{2}$ The NovaVision website features anecdotal case vignettes of patients who experienced huge recovery from scotomas, hardly representative of the mean $2.5^{\circ}-4.9^{\circ}$ improvement reported by Sabel in his studies. ${ }^{3}$ The website also announces that NovaVision has raised \$20 million in venture capital funds to finance its expansion into the US marketplace. Sabel's financial stake is undisclosed in all his publications.

The saga of Sabel's visual restoration therapy provides a cautionary tale. An investigator proposes a new therapy for a condition that has no treatment. He adduces supporting evidence by carrying out a number of trials, but fails to control properly for a source of artefact. Meanwhile, he launches a commercial venture, based on his own research, and becomes financially involved. When his data are challenged, he agrees to an independent test of his therapy in collaboration with a third party. When the results prove him wrong, he rejects them. Meanwhile, trusting patients continue to sign up for the treatment programme, motivated by hope and the knowledge that nothing else is available. 
Br J Ophthalmol 2005;89:792-794.

doi: 10.1136/bjo.2005.072967

Correspondence to: J C Horton, UCSF, San Francisco, CA 94143, USA

hortonj@vision.ucsf.edu

\section{REFERENCES}

1 Reinhard J, Schreiber A, Schiefer U, et al. Does visual restitution training change absolute homonymous visual field defects? A fundus controlled study. Br J Ophthalmol 2005;89:30-5.

2 Sabel BA, Kenkel S, Kasten E. Vision restoration therapy. Br J Ophthalmol 2005;89:521-4.

3 Kasten E, Wust S, Behrens-Baumann W, et al. Computer-based training for the treatment of partial blindness. Nat Med 1998;4:1083-7.
4 Kasten E, Wuest S, Sabel BA. Residual vision in transition zones in patients with cerebral blindness. J Clin Exp Neuropsychol 1998;20:581-98.

5 Kasten E, Poggel DA, Muller-Oehring E, et al. Restoration of vision II: Residual functions and training-induced visual field enlargement in braindamaged patients. Restor Neurol Neurosci 1999:15:273-87.

6 Sabel BA, Kasten E. Restoration of vision by training of residual functions. Curr Opin Ophthalmol 2000;11:430-6.

7 Kasten E, Muller-Oehring E, Sabel BA. Stability of visual field enlargements following computerbased restitution training - results of a follow-up. J Clin Exp Neuropsychol 2001 ;23:297-305.

8 Mueller I, Poggel DA, Kenkel S, et al. Vision restoration therapy after brain damage: Subjective improvements of activities of daily life and their relationship to visual field enlargements Visual Impairment Research 2003;5:157-78.

9 Sabel BA, Kenkel S, Kasten E. Vision restoration therapy (VRT) efficacy as assessed by comparitive perimetric analysis and subjective questionnaires. Restor Neurol Neurosci 2005; (in press).

10 Horton JC. Disappointing results from NovaVision's visual restoration therapy. Br J Ophthalmol 2005;89:1-2.

11 Heijl A, Bengtsson B. The effect of perimetric experience in patients with glaucoma. Arch Ophthalmol 1996:114:19-22.

12 Gilbert CD, Wiesel TN. Receptive field dynamics in adult primary visual cortex. Nature 1992;356:150-2.

13 Kaas JH, Krubitzer LA, Chino YM, et al. Reorganization of retinotopic cortical maps in adult mammals after lesions of the retina. Science 1990;248:229-31 